Review Article

\title{
Melatonin Exerts Anti-Inflammatory, Antioxidant, and Neuromodulatory Effects That Could Potentially Be Useful in the Treatment of Vertigo
}

\author{
Joaquin Guerra $\mathbb{D}^{1}$ and Jesus Devesa $\mathbb{D}^{2}$ \\ ${ }^{1}$ Otolaryngology, Foltra Medical Center, Travesía Montouto 24, Teo 15886, Spain \\ ${ }^{2}$ Scientific Direction, Foltra Medical Center, Teo 15886, Spain \\ Correspondence should be addressed to Joaquin Guerra; joaquin.guerra.otorrino@gmail.com
}

Received 5 December 2020; Revised 10 March 2021; Accepted 15 March 2021; Published 23 March 2021

Academic Editor: Johan H.M. Frijns

Copyright (c) 2021 Joaquin Guerra and Jesus Devesa. This is an open access article distributed under the Creative Commons Attribution License, which permits unrestricted use, distribution, and reproduction in any medium, provided the original work is properly cited.

\begin{abstract}
The acute phase of vertigo involves multiple neurotransmitters, inflammatory mediators, and products of oxidative stress. The vestibular pathway has multiple melatonin receptors distributed along its path, both centrally and peripherally. In addition, melatonin has been shown to be a powerful antioxidant and anti-inflammatory agent against factors related to vertigo, such as Bax/caspases, interleukins, and chemokines. Likewise, it exerts central GABAergic, antidopaminergic, and anti-migraine functions and regulates sympathetic activity in a similar way to the drugs classically used in acute vestibular crisis. In this review, the role of melatonin as a potential treatment of the acute phase of vertigo is discussed.
\end{abstract}

\section{Introduction}

The acute phase of vertigo appears in several vestibular syndromes with different pathophysiology, such as Menière's disease (MD), vestibular neuritis (VN), vestibular migraine (VM), and benign paroxysmal positional vertigo (BPPV). Due to the involvement of the inner ear, the symptoms may include not only recurrent attacks of vertigo but also fluctuating hearing loss or tinnitus. In addition, multiple central nervous system conditions associated with migraine or cerebrovascular and neurodegenerative disorders have been described, which can manifest vertiginous attacks $[1,2]$.

Vestibular syndromes imply that metabolic factors may act in their development, such as (1) multiple neurotransmitters with different effects: excitatory (glutamate, dopamine, and serotonin), modulating (histamine and enkephalins), or inhibitory (GABA and glycine); (2) inflammatory cytokines (TNF and IL3); (3) reactive oxygen species (ROS); and (4) other factors [3-5].

Although the different types of vertigo imply a different pathophysiology, the treatment of the acute crisis is usually symptomatic, which implies a similar pharmacological management, which generally acts by exerting a sedative effect. For this reason, among others, antihistamines, anticholinergics, benzodiazepines, or antidopaminergic drugs are used [3]. However, considering that the incidence of vertiginous syndrome is particularly higher in the elderly population, in this and other risk groups, the use of a lower dose of the aforementioned drugs or the use of other treatments with fewer side effects could be useful [6].

That is why in this study, we will analyze the possible role that melatonin, a harmless hormone, could play in regulating the acute phase of vertigo.

\section{Melatonin and the Vestibular Pathway}

Melatonin performs extensive functions not only in the inner ear but also in the vestibular pathway, regulating its function. Precisely, in the inner ear, there is a wide and diffuse expression of melatonin MT1 receptors, distributed in many structures, including the organ of Corti, the spiral and vestibular ganglion, vestibular sensory cells, dark 
vestibular cells, transitional cells, or epithelial cells of the endolymphatic sac [7]. With respect to the central vestibular structures, MT1 and MT2 receptors are also found in the vestibular nuclei, the thalamic vestibular pathway, and the cerebral and cerebellar cortex [8]. The cerebellum expresses the highest number of melatoninergic receptors [9]. Clinically, melatonin receptors found in the area postrema, a structure in the medulla oblongata of the brainstem, can modulate vomiting and other sympathetic responses that characterize the attack of vertigo [10]. The paraventricular nuclei and the reuniens connect with the limbic system, and this may be the reason for their regulation in mood and sedation, relevant elements for the control of vertigo [11].

Furthermore, several reports have attempted to explain the disorganization of circadian rhythms in patients with vestibular disorders. For example, aging implies a degeneration of the vestibular pathway, in which the dysregulation of the circadian rhythm could influence the genesis of vestibular imbalance [12]. Furthermore, melatonin has been proposed as a prophylactic agent in the prevention of migraine attacks, a condition that can be associated with vertigo $[1,2,13]$. In addition, in patients with bilateral vestibular loss, there is a lack of synchronization between temperature and the rest-activity cycle, which affects the physiology of melatonin regulation [14]. Although the effect of melatonin can be exerted by direct action, it is true that it has the potential to modulate other compounds, enhancing or inhibiting them, and thus their actions $[15,16]$.

\section{Role of Melatonin as an Anti-Inflammatory and Antioxidant Vestibular Agent}

Vertigo patients show higher levels of reactive oxygen species (ROS) and superoxide metabolites than healthy subjects, as shown by multiple reports from subjects with different vestibular syndromes, such as BPPV, MD, or unspecified situations of chronic subjective vertigo. It includes higher levels of hydrogen peroxide, oxidation products of thiol and other ROS, and lower activity of superoxide dismutase (SOD), glutathione content, and catalase [17-21]. Oxidative stress may be due to the physiological stress that vertigo induces [18]. The antioxidant effect of melatonin is well known, since it acts as a direct scavenger of free radicals with the ability to detoxify both reactive oxygen and reactive nitrogen species [22]. In animal models, the otoprotective function of melatonin has been demonstrated after exposure to gentamicin in the inner ear; this otoprotection is mainly based on the inhibition of the genesis of free radicals or scavenging them. Gentamicin induces an increase in the levels of ROS and proapoptotic Bcl-2-associated protein $X(\mathrm{Bax})$ in utricular hair cells, in turn inhibiting the expression of B-cell lymphoma 2 (Bcl-2). Melatonin reverses this event by inhibiting the expression of caspase-3. This protein is essential in the activation of programmed cell death [23].

Interestingly, in patients with chronic subjective dizziness, an inflammatory response with elevated serum levels of tumor necrosis factor $\alpha$ (TNF) and interferon $\gamma$ (IFN $\gamma$ ) has been reported [20]. Along with similar lines, patients with
MD show an elevation of various interleukins (IL- $1 \beta$, IL1RA, and IL-6) and TNF baseline levels. Furthermore, in these patients, the two subgroups can be differentiated according to their IL-1 $\beta$ profile; those with higher basal levels exhibit increased levels of cytokines and chemokines (CCLs). Interestingly, the proinflammatory immune response appears to increase in those subjects exposed to allergenic extracts of Aspergillus and Penicillium involving TNF, which points to a possible allergic association [24].

According to these reports, the levels of IL- $1 \beta$, CCL3, CCL22, and CXCL1 have been proposed as differentiating markers of MD from other vestibular syndromes that can confuse the diagnosis, such as VM, whose clinical expression can be very similar [25]. In VN, the CD40 receptor, which belongs to the family of TNF, and its ligand (CD40L) have been suggested to be involved in the progression and genesis of the disease, thus increasing the production of several proinflammatory cytokines, such as TNF [26]. As described above, vestibular syndromes exhibit inflammatory reactions during acute attacks and subjects with chronic vertigo have higher basal levels of inflammatory mediators, so that melatonin theoretically would be able to regulate not only attacks but also recurrences, given its regulation of the release of various cytokines. Although no report has specifically focused on the role of melatonin in the vestibular system and these cytokines, this hormone could centrally or peripherally control the levels of CCLs, ILs, and TNF [27]. For example, while central IL-1 $\beta$ suppresses the physiological nocturnal secretion of melatonin [28], exogenous administration of this hormone reduces the inflammatory activity of IL-1 $\beta$ [29] by upregulating the expression of superoxide dismutase (SOD) and downregulating the expression of Bax [30]. Inhibition of IL-1 $\beta$ and TNF has also been reported to downregulate PI3K/AKT, ERK, NF- $\kappa \mathrm{B}$ signaling pathways, as well as overexpression of miR-3150a$3 p$ [31]. Other studies show that melatonin inhibits the gene expression of other cytokines or their receptors, such as IL1RA, or the expression of CCL induced by LPS in different inflammatory conditions [32, 33].

As shown in Figure 1, melatonin may exert beneficial effects by blocking the activity of vestibular oxidative and inflammatory stress through several pathways.

\section{Melatonin as a Modulator in the Vestibular Neurotransmission}

Gamma-aminobutyric acid (GABA) is the predominant inhibitory neurotransmitter in the vestibular pathway. Of the three GABA receptors described, GABA-A and GABA-B are involved in vestibular neurotransmission [34]. Studies show that GABA plays a plausible role in inner ear afferent transmission, but its role as the primary transmitter at this level is unclear. It is accepted that its function is to modulate neuronal transmission, through the presynaptic inhibition of $\mathrm{Ca}^{2+}$ channels and/or the activation of $\mathrm{Cl}$ channels. Therefore, it can indirectly decrease the release of presynaptic neurotransmitters to affect the excitability of postsynaptic cells $[35,36]$. The central vestibular nuclei receive inhibitory inputs that are mediated by GABA-A and GABA- 


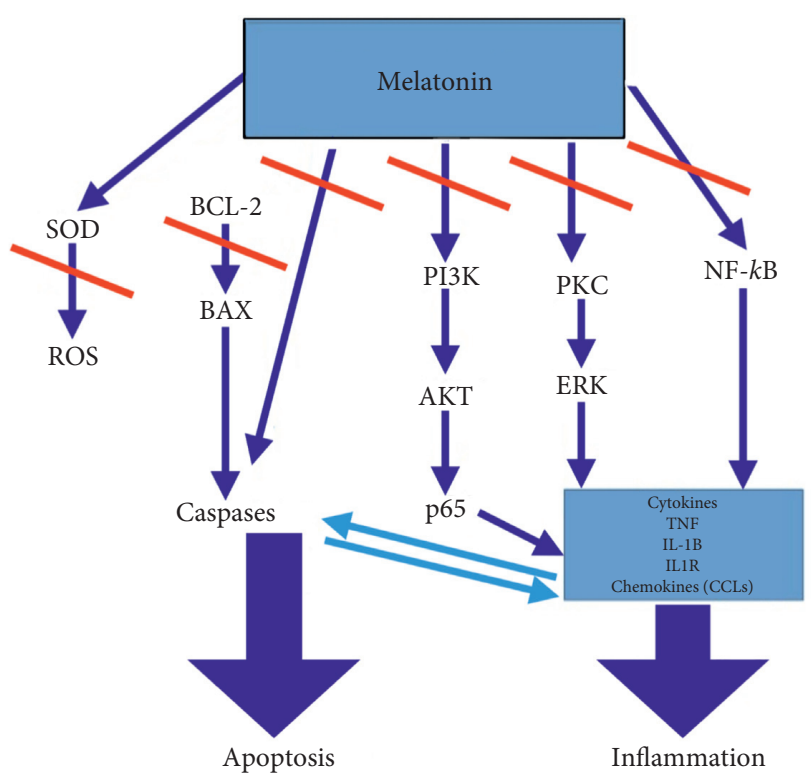

Figure 1: Potential anti-inflammatory and antioxidant actions of melatonin in vestibular disorders. Blue arrows indicate stimulation or activation of some enzymes, signaling pathways, inflammation markers, and genes, while the red arrows indicate the inhibitory effects of melatonin on these pathways, thus inhibiting apoptosis and inflammation. Note how melatonin activates SOD.

$B$ receptors. These GABA-A inputs arise primarily from the commissural fibers of the vestibular nuclei and the cerebellum [37]. Theoretically, the treatment with agonists of the GABA-A (benzodiazepines) and GABA-B (baclofen) receptors is based on an effect on the central vestibular sensory pathways [38]. Melatonin can also regulate the GABAergic synaptic transmission and thus modulates the activity of its receptor [39]. Its sedative effect is mainly enabled by binding to the GABA-A receptor, as it occurs with benzodiazepines [40]. This sedative action may induce a decrease in blood pressure [41]. In rats submitted to pinealectomy, the interruption of the cerebral circadian rhythm of this neurotransmitter prevents the union of benzodiazepines to their receptors; with the use of exogenous melatonin, this phenomenon is reversed [42]. In vitro studies have also shown that melatonin increases the amplitude and frequency of miniature postsynaptic current inhibitory chemicals (mIPSCs), enhancing the hippocampal GABAergic transmission [43].

Several findings support a possible involvement of dopamine as a modulator of excitatory vestibular neurotransmission in the postsynaptic afferent terminals in at least 2 of the 5 dopamine receptors identified. In the vestibular neuroepithelium of mammals, immunochemical tests show that D1 and D2 receptors (coupled to $G$ proteins) are expressed in the vestibular hair cell membranes [44]. The responses of these receptors not only modulate postsynaptic glutamate receptors but may also have a protective function on vestibular dendrites [45]. The existence of dopamine D2 receptors has been reported in the vestibular nuclei [46]. The use of antidopaminergic drugs (sulpiride and prochlorperazine) exerts a modulating effect on vestibular neurons and controls vomit $[3,47]$. Although there is no report directly involving melatonin in this effect in vestibular structures, it has been demonstrated that this hormone modulates dopamine and can inhibit its release in specific areas in the CNS of mammals, such as the hypothalamus, hippocampus, striatum, medulla-pons, and retina [48].

Other compounds involved in vestibular neurochemistry, such as substance $\mathrm{P}$ or calcitonin gene-related peptide (CGRP), both implicated in migraine, and thus potentially vestibular migraine (VM), are also inhibited by melatonin [49-51]. Furthermore, TNF stimulates CGRP transcription, whereas as previously described, melatonin is capable of inhibiting TNF release [31, 50]. CGRP antagonists are currently being developed for the treatment of migraine, although they should not be considered as first-line treatments [52].

Table 1 summarizes the potential effects of melatonin on neurotransmitters presumably involved in the genesis of vertigo.

\section{Regulation of Melatonin in the Vestibular Sympathetic Activity}

Melatonin release is controlled by the sympathetic innervation of the pineal gland, which mediates the inhibitory effect of light on pineal melatonin secretion. This pathway begins in the retina, influencing the biological clock of the suprachiasmatic nucleus [53], and then inhibits the paraventricular nucleus and interrupts the stimulation of the intermediolateral nucleus, inducing melatonin synthesis [54]. As previously described, the physiology of melatonin is closely related to the sympathetic nervous system and sympathetic activity correlates with circadian rhythms [55]. For example, the cardiovascular response to laryngoscopy and laryngotracheal intubation is attenuated by the administration of melatonin [41].

Patients diagnosed with vertigo show less parasympathetic activity; the ratio of sympathetic/parasympathetic activity is higher than in healthy subjects [18]. The effects of melatonin on the autonomic system cause a reduction in the adrenergic flow [56] and induce relaxation of the smooth muscle of the arterial wall by increasing the availability of nitric oxide [57]. Furthermore, melatonin is capable of lowering blood pressure, specifically binding to its MT1 and MT2 receptors in blood vessels, thus blocking the catecholaminergic response [58].

Pinealectomized rats show higher circulating levels of catecholamines when stimulated with IL1- $\beta$, an inflammatory mediator involved in vertigo, as mentioned previously [24]. This effect is reverted after intraventricular infusion of melatonin [59]. In spontaneously hypertensive rats, acute administration of melatonin lowered blood pressure along with norepinephrine levels [60], although the authors found that this effect might not be mediated by melatonin receptors or $\alpha$-adrenoceptors, but by the antioxidant effect of melatonin that inhibits inositol phosphate [61]. In humans, exogenous use of melatonin has been shown to be effective in reducing circulating catecholamine levels [62], as well as 
TABLE 1: Melatonin effects on neurotransmitters.

\begin{tabular}{|c|c|c|c|}
\hline Neurotransmitter & Vestibular receptors & Melatonin action & Effect \\
\hline GABA & $\begin{array}{l}\text { GABA-A } \\
\text { GABA-B }\end{array}$ & Stimulation & $\begin{array}{c}\text { Sedative } \\
\text { Anxiolytic }\end{array}$ \\
\hline Dopamine & $\begin{array}{l}\text { D1 } \\
\text { D2 }\end{array}$ & Inhibition & $\begin{array}{c}\text { Sedative } \\
\text { Antiemetic }\end{array}$ \\
\hline Substance P & NK1R & Inhibition & $\begin{array}{c}\text { Anti-inflammatory } \\
\text { Anti-migraine }\end{array}$ \\
\hline CGRP & CALCRL & Inhibition & $\begin{array}{c}\text { Anti-inflammatory } \\
\text { Anti-migraine }\end{array}$ \\
\hline
\end{tabular}

blood pressure, carotid pulsatility index [63], and sympathetic nerve responses to orthostatic stress [64].

\section{Conclusions}

Based on the data included in this review, it seems obvious that the use of melatonin in the acute phase of vertigo can be highly effective, although more studies and clinical trials are needed. However, despite the fact that the effect in humans may be more limited than in laboratory animals, it is evident that the adjuvant use of melatonin with other drugs could not only improve the vestibular symptoms of acute vertigo crisis but also prevent the increase of doses of commonly used drugs with the consequent increase in pharmacological toxicity. This type of combined treatment would be especially indicated in risk groups, such as the elderly population. Moreover, melatonin is a practically harmless hormone; the lethal dose 50 could not be found yet. Paradoxically, some reports showed transient dizziness as a side effect. This symptom may be only a subjective report or it may be associated with its sedative function, and it does not limit its use as with other drugs employed in acute vertigo [65]. However, we cannot ignore the possibility of undesirable effects appearing in patients who are recovering after an attack of acute vertigo, delaying vestibular compensation. These adverse effects have been observed in posturographic results and in oculomotor tests, with a decrease in saccade accuracy or smooth pursuit gain [66]. Furthermore, the decreased sympathetic response may theoretically exert a greater intolerance to orthostatism, although this conclusion may be questionable [64]. Moreover, there are no reports of vestibular worsening demonstrated in neurophysiological tests, such as vestibular evoked myogenic potential (VEMP) [67]. As previously described, its safety profile, even at extremely high doses, is wide [65].

Although systemic administration is safe and favors effects on different organs of the vestibular pathway, it remains to be seen whether topical (transtympanic) administration could be effective for pathologies of peripheral origin. A route of entry for various metabolites with oxidizing or inflammatory power is the round window. The main advantage of this approach relies on the fact that melatonin would perfuse directly to the inner ear, as it occurs with the intratympanic corticosteroid treatment. Moreover, treating melatonin topically could minimize the effect of mediators that access through this route of entry, implied in the development of vestibular syndromes such as labyrinthitis or endolymphatic hydrops [68]. In conclusion melatonin administration in vertigo could be a new therapeutic effect of melatonin, among the many already described that this hormone exerts in human pathologies.

\section{Data Availability}

No data are used to support this study.

\section{Conflicts of Interest}

The authors declare no conflicts of interest.

\section{References}

[1] C. Agrup, M. Gleeson, and P. Rudge, "The inner ear and the neurologist," Journal of Neurology, Neurosurgery \& Psychiatry, vol. 78, no. 2, pp. 114-122, 2007.

[2] T. L. Thopson and R. V. Amedee, "A review of common peripheral and central vestibular disorders," Ochsner Journal, vol. 9, pp. 20-26, 2009.

[3] E. Soto and R. Vega, "Neuropharmacology of vestibular system disorders," Current Neuropharmacology, vol. 8, no. 1, pp. 26-40, 2010.

[4] C. Kao, K. Tsai, Y. Cheng, C. Kuo, S. Lee, and R. Chan, "Vestibular rehabilitation ameliorates chronic dizziness through the SIRT1 axis," Frontiers in Aging Neuroscience, vol. 6, p. 27, 2014.

[5] J. Guerra and R. Cacabelos, "Pharmacoepigenetics of vertigo and related vestibular syndromes," in Translational Epigenetics, Pharmacoepigenetics, R. Cacabelos, Ed., vol. 10, pp. 755-779, Academic Press, Cambridge, MA, USA, 1st edition, 2019.

[6] J. Rushabh and A. S. Dagli, "Polypharmacy: a global risk factor for elderly people," Journal of International Oral Health, vol. 6, 2014.

[7] M. Takumida and M. Anniko, "Localization of melatonin and its receptors (melatonin $1 \mathrm{a}$ and $1 \mathrm{~b}$ receptors) in the mouse inner ear," Acta Oto-Laryngologica, vol. 139, no. 11, pp. 948-952, 2019.

[8] K. Y. Ng, M. K. Leong, H. Liang, and G. Paxinos, "Melatonin receptors: distribution in mammalian brain and their respective putative functions," Brain Structure and Function, vol. 222, no. 7, pp. 2921-2939, 2017.

[9] C. Mazzucchelli, M. Pannacci, R. Nonno, V. Lucini, F. Fraschini, and B. Michaylov Stankov, "The melatonin receptor in the human brain: cloning experiments and distribution studies," Molecular Brain Research, vol. 39, no. 1-2, pp. 117-126, 1996. 
[10] A. D. Miller and R. A. Leslie, "The area postrema and vomiting," Frontiers in Neuroendocrinology, vol. 15, no. 4, pp. 301-320, 1994.

[11] V. Colavito, C. Tesoriero, A. T. Wirtu, G. Grassi-Zucconi, and M. Bentivoglio, "Limbic thalamus and state-dependent behavior: the paraventricular nucleus of the thalamic midline as a node in circadian timing and sleep/wake-regulatory networks," Neuroscience \& Biobehavioral Reviews, vol. 54, pp. 3-17, 2015.

[12] D. Alpini, A. Cesarani, F. Fraschini, R. Kohen-Raz, S. Capobianco, and F. Cornelio, "Aging and vestibular system: specific tests and role of melatonin in cognitive involvement," Archives of Gerontology and Geriatrics, vol. 38, pp. 13-25, 2004.

[13] R. Long, Y. Zhu, and S. Zhou, "Therapeutic role of melatonin in migraine prophylaxis," Medicine, vol. 98, Article ID e14099, 2019.

[14] T. Martin, S. Moussay, I. Bulla et al., "Exploration of circadian rhythms in patients with bilateral vestibular loss," PLoS One, vol. 11, Article ID e0155067, 2016.

[15] M. Banach, E. Gurdziel, M. Jędrych, and K. K. Borowicz, "Melatonin in experimental seizures and epilepsy," Pharmacological Reports, vol. 63, no. 1, pp. 1-11, 2011.

[16] R. Hardeland, "Melatonin and inflammation-Story of a double-edged blade," Journal of Pineal Research, vol. 65, Article ID e12525, 2018.

[17] F. Goto, R. Ide, H. Yabe, T. Kunihiro, and K. Ogawa, "Oxidative stress and Ménière's disease and benign paroxysmal positional vertigo," Equilibrium Research, vol. 68, no. 1, pp. 9-13, 2009.

[18] K. Ohara, Y. Inoue, Y. Sumi et al., "Oxidative stress and heart rate variability in patients with vertigo," Acute Medicine \& Surgery, vol. 2, no. 3, pp. 163-168, 2014.

[19] E. Sahin, I. Deveci, I. Deveci et al., "Oxidative status in patients with benign paroxysmal positional vertigo," The Journal of International Advanced Otology, vol. 14, no. 2, pp. 299-303, 2018.

[20] Z. Fang, K. Huang, C. Gil, J. Jeong, H. Yoo, and H. Kim, "Biomarkers of oxidative stress and endogenous antioxidants for patients with chronic subjective dizziness," Science Reports, vol. 10, p. 1478, 2020.

[21] J. Li, R. Wu, B. Xia, X. Wang, and M. Xue, "Serum levels of superoxide dismutases in patients with benign paroxysmal positional vertigo," Bioscience Reports, vol. 40, 2020.

[22] R. J. Reiter, D.-X. Tan, L. C. Manchester, and W. Qi, "Biochemical reactivity of melatonin with reactive oxygen and nitrogen species: a review of the evidence," Cell Biochemistry and Biophysics, vol. 34, no. 2, pp. 237-256, 2001.

[23] J.-B. Kim, J. Y. Jung, J.-C. Ahn, C. K. Rhee, and H.-J. Hwang, "Antioxidant and anti-apoptotic effect of melatonin on the vestibular hair cells of rat utricles," Clinical and Experimental Otorhinolaryngology, vol. 2, no. 1, p. 6, 2009.

[24] L. Frejo, A. Gallego-Martinez, T. Requena et al., "Proinflammatory cytokines and response to molds in mononuclear cells of patients with Meniere disease," Science Reports, vol. 8, p. $5974,2018$.

[25] M. Flook, L. Frejo, A. Gallego-Martinez et al., "Differential proinflammatory signature in vestibular migraine and meniere disease," Frontiers in Immunology, vol. 10, p. 1229, 2019.

[26] A. Greco, G. F. Macri, A. Gallo et al., "Is vestibular neuritis an immune related vestibular neuropathy inducing vertigo?" Journal of Immunology Research, vol. 2014, pp. 1-8, 2014.
[27] M. Zarezadeh, M. Khorshidi, M. Emami et al., "Melatonin supplementation and pro-inflammatory mediators: a systematic review and meta-analysis of clinical trials," European Journal of Nutrition, vol. 59, no. 5, pp. 1803-1813, 2019.

[28] A. P. Herman, J. Bochenek, K. Król et al., "Central interleukin$1 \beta$ suppresses the nocturnal secretion of melatonin," Mediators of Inflammation, vol. 2016, pp. 1-15, 2016.

[29] G. Mannino, F. Caradonna, I. Cruciata, A. Lauria, A. Perrone, and C. Gentile, "Melatonin reduces inflammatory response in human intestinal epithelial cells stimulated by interleukin$1 \beta$," Journal of Pineal Research, vol. 67, Article ID e12598, 2019.

[30] X. Liu, Y. Gong, K. Xiong et al., "Melatonin mediates protective effects on inflammatory response induced by interleukin-1 beta in human mesenchymal stem cells," Journal of Pineal Research, vol. 55, no. 1, pp. 14-25, 2013.

[31] C. Huang, C. Chiou, S. Liu et al., "Melatonin attenuates TNF$\alpha$ and IL- $1 \beta$ expression in synovial fibroblasts and diminishes cartilage degradation: implications for the treatment of rheumatoid arthritis," Journal of Pineal Research, vol. 66, Article ID e12560, 2019.

[32] H. J. Park, H. J. Kim, J. Ra et al., "Melatonin inhibits lipopolysaccharide-induced CC chemokine subfamily gene expression in human peripheral blood mononuclear cells in a microarray analysis," Journal of Pineal Research, vol. 43, no. 2, pp. 121-129, 2007.

[33] G. Yu, H. Kubota, M. Okita, and T. Maeda, "The anti-inflammatory and antioxidant effects of melatonin on LPSstimulated bovine mammary epithelial cells," PLoS One, vol. 12, Article ID e0178525, 2017.

[34] C. Gliddon, C. Darlington, and P. Smith, "GABAergic systems in the vestibular nucleus and their contribution to vestibular compensation," Progress in Neurobiology, vol. 75, no. 1, pp. 53-81, 2005.

[35] A. E. Herbison and S. M. Moenter, "Depolarising and hyperpolarising actions of GABAA receptor activation on gonadotrophin-releasing hormone neurones: towards an emerging consensus," Journal of Neuroendocrinology, vol. 23, no. 7, pp. 557-569, 2011.

[36] S. Geisler, C. L. Schöpf, R. Stanika et al., "Presynaptic $\alpha 2 \delta-2$ calcium channel subunits regulate postsynaptic gabaa receptor abundance and axonal wiring," The Journal of Neuroscience, vol. 39, no. 14, pp. 2581-2605, 2019.

[37] S. M. Highstein and G. R. Holstein, "The anatomy of the vestibular nuclei," Progress in Brain Research, vol. 151, pp. 157-203, 2006.

[38] M. Strupp, M. J. Thurtell, A. G. Shaikh, T. Brandt, D. S. Zee, and R. J. Leigh, "Pharmacotherapy of vestibular and ocular motor disorders, including nystagmus," Journal of Neurology, vol. 258, no. 7, pp. 1207-1222, 2011.

[39] L. P. Nilcs, D. S. Pickering, and M. A. Arciszewski, "Effects of chronic melatonin administration on GABA and diazepam binding in rat brain," Journal of Neural Transmission, vol. 70, no. 1-2, pp. 117-124, 1987.

[40] Q. Wan, H.-Y. Man, F. Liu et al., "Differential modulation of GABAA receptor function by Mel1a and Mel1b receptors," Nature Neuroscience, vol. 2, no. 5, pp. 401-403, 1999.

[41] P. Gupta, D. Jethava, R. Choudhary, and D. Jethava, "Role of melatonin in attenuation of haemodynamic responses to laryngoscopy and intubation," Indian Journal of Anaesthesia, vol. 60 , no. 10 , p. 712, 2016.

[42] R. E. Rosenstein and D. P. Cardinali, "Central GABAergic mechanisms as targets for melatonin activity in brain," 
Neurochemistry International, vol. 17, no. 3, pp. 373-379, 1990.

[43] X.-P. Cheng, H. Sun, Z.-Y. Ye, and J.-N. Zhou, "Melatonin modulates the GABAergic response in cultured rat hippocampal neurons," Journal of Pharmacological Sciences, vol. 119, no. 2, pp. 177-185, 2012.

[44] M. J. Drescher, W. J. Cho, A. J. Folbe et al., “An adenylyl cyclase signaling pathway predicts direct dopaminergic input to vestibular hair cells," Neuroscience, vol. 171, no. 4, pp. 1054-1074, 2010.

[45] G. N. Andrianov, I. V. Ryzhova, and T. V. Tobias, "Dopaminergic modulation of afferent synaptic transmission in the semicircular canals of frogs," Neurosignals, vol. 17, no. 3, pp. 222-228, 2009.

[46] N. Vibert, M. Serafin, O. Crambes, P.-P. Vida, and M. Mühlethaler, "Dopaminergic agonists have both presynaptic and postsynaptic effects on the Guinea-pig's medial vestibular nucleus neurons," European Journal of Neuroscience, vol. 7, no. 4, pp. 555-562, 1995.

[47] J. M. Espinosa-Sanchez and J. A. Lopez-Escamez, "The pharmacological management of vertigo in Meniere disease," Expert Opinion on Pharmacotherapy, vol. 21, no. 14, pp. 1753-1763, 2020.

[48] N. Zisapel, "Melatonin-dopamine interactions: from basic neurochemistry to a clinical setting Cell," Cellular and Molecular Neurobiology, vol. 21, no. 6, pp. 605-616, 2001.

[49] M. Juszczak and B. Stempniak, "Melatonin inhibits the substance P-induced secretion of vasopressin and oxytocin from the rat hypothalamo-neurohypophysial system: in vitro studies," Brain Research Bulletin, vol. 59, no. 5, pp. 393-397, 2003.

[50] P. L. Durham, "Calcitonin gene-related peptide (CGRP) and migraine," Headache: The Journal of Head and Face Pain, vol. 46, no. s1, pp. S3-S8, 2006.

[51] M. Ansari, A. Karkhaneh, A. Kheirollahi, S. Emamgholipour, and M. H. Rafiee, "The effect of melatonin on gene expression of calcitonin gene-related peptide and some proinflammatory mediators in patients with pure menstrual migraine," Acta Neurologica Belgica, vol. 117, no. 3, pp. 677-685, 2017.

[52] M. Deen, E. Correnti, K. Kamm et al., "Blocking CGRP in migraine patients - a review of pros and cons," Journal of Headache Pain, vol. 18, p. 96, 2017.

[53] S. Perreau-Lenz, A. Kalsbeek, M.-L. Garidou et al., "Suprachiasmatic control of melatonin synthesis in rats: inhibitory and stimulatory mechanisms," European Journal of Neuroscience, vol. 17, no. 2, pp. 221-228, 2003.

[54] L. Smale, V. M. Cassone, R. Y. Moore, and L. P. Morin, "Paraventricular nucleus projections mediating pineal melatonin and gonadal responses to photoperiod in the hamster," Brain Research Bulletin, vol. 22, no. 2, pp. 263-269, 1989.

[55] P. Boudreau, G. Dumont, N. Kin, C. Walker, and D. Boivin, "Correlation of heart rate variability and circadian markers in humans," Annual International Conference of the IEEE Engineering in Medicine and Biology Society 2011, vol. 2011, pp. 681-682, 2011.

[56] M. M. Anwar, A.-R. M. A. Meki, and H. H. Abu Rahma, "Inhibitory effects of melatonin on vascular reactivity: possible role of vasoactive mediators," Comparative Biochemistry and Physiology Part C: Toxicology \& Pharmacology, vol. 130, no. 3, pp. 357-367, 2001.

[57] P. Shukla, C. Sun, and S. T. O'Rourke, "Melatonin inhibits nitric oxide signaling by increasing PDE5 phosphorylation in coronary arteries," American Journal of Physiology-Heart and
Circulatory Physiology, vol. 303, no. 12, pp. H1418-H1425, 2012.

[58] J. Baker and K. Kimpinski, "Role of melatonin in blood pressure regulation: an adjunct anti-hypertensive agent," Clinical and Experimental Pharmacology and Physiology, vol. 45, no. 8, pp. 755-766, 2018.

[59] M. Wang, K. Yokotani, K. Nakamura, Y. Murakami, S. Okada, and Y. Osumi, "Melatonin inhibits the central sympathoadrenomedullary outflow in rats," The Japanese Journal of Pharmacology, vol. 81, no. 1, pp. 29-33, 1999.

[60] K. Laflamme, L. Wu, S. Foucart, and J. Champlain, "Impaired basal sympathetic tone and $\alpha 1$-adrenergic responsiveness in association with the hypotensive effect of melatonin in spontaneously hypertensive rats," American Journal of $\mathrm{Hy}$ pertension, vol. 11, pp. 219-229, 1998.

[61] L. Wu, R. Wang, and J. D. Champlain, "Enhanced inhibition by melatonin of $\alpha$-adrenoceptor- induced aortic contraction and inositol phosphate production in vascular smooth muscle cells from spontaneously hypertensive rats," Journal of $\mathrm{Hy}$ pertension, vol. 16, no. 3, pp. 339-347, 1998.

[62] S. Arangino, A. Cagnacci, M. Angiolucci et al., "Effects of melatonin on vascular reactivity, catecholamine levels, and blood pressure in healthy men," The American Journal of Cardiology, vol. 83, no. 9, pp. 1417-1419, 1999.

[63] A. Cagnacci, S. Arangino, M. Angiolucci, E. Maschio, G. Longu, and G. B. Metis, "Potentially beneficial cardiovascular effects of melatonin administration in women," Journal of Pineal Research, vol. 22, no. 1, pp. 16-19, 1997.

[64] C. A. Ray, "Melatonin attenuates the sympathetic nerve responses to orthostatic stress in humans," The Journal of Physiology, vol. 551, no. 3, pp. 1043-1048, 2003.

[65] R. M. L. Colunga Biancatelli, M. Berrill, Y. H. Mohammed, and P. E. Marik, "Melatonin for the treatment of sepsis: the scientific rationale," Journal of Thoracic Disease, vol. 12, no. S1, pp. S54-S65, 2020.

[66] F. Fraschini, A. Cesarani, D. Alpini, D. Esposti, and B. M. Stankov, "Melatonin influences human balance," Neurosignals, vol. 8, no. 1-2, pp. 111-119, 1999.

[67] J. S. Cook and C. A. Ray, "Melatonin attenuates the vestibulosympathetic but not vestibulocollic reflexes in humans: selective impairment of the utricles," Journal of Applied Physiology, vol. 109, no. 6, pp. 1697-1701, 2010.

[68] S. Cureoglu, P. A. Schachern, A. Rinaldo, V. Tsuprun, A. Ferlito, and M. M. Paparella, "Round window membrane and labyrinthine pathological changes: an overview," Acta Oto-Laryngologica, vol. 125, no. 1, pp. 9-15, 2005. 